\title{
GRAVITATIONAL-WAVE CONSTRAINTS ON THE PROGENITORS OF FAST RADIO BURSTS
}

Thomas Callister, Jonah Kanner, and Alan Weinstein

LIGO Laboratory, California Institute of Technology, Pasadena, CA 91125, USA; tcallist@ caltech.edu Received 2016 April 7; revised 2016 June 11; accepted 2016 June 14; published 2016 June 30

\begin{abstract}
The nature of fast radio bursts (FRBs) remains enigmatic. Highly energetic radio pulses of millisecond duration, FRBs are observed with dispersion measures consistent with an extragalactic source. A variety of models have been proposed to explain their origin. One popular class of theorized FRB progenitor is the coalescence of compact binaries composed of neutron stars and/or black holes. Such coalescence events are strong gravitational-wave emitters. We demonstrate that measurements made by the LIGO and Virgo gravitational-wave observatories can be leveraged to severely constrain the validity of FRB binary coalescence models. Existing measurements constrain the binary black hole rate to approximately 5\% of the FRB rate, and results from Advanced LIGO's O1 and O2 observing runs may place similarly strong constraints on the fraction of FRBs due to binary neutron star and neutron star-black hole progenitors.
\end{abstract}

Key words: black hole physics - gravitation - gravitational waves - stars: black holes - stars: neutron

\section{INTRODUCTION}

Recent years have seen the emergence of fast radio bursts (FRBs) as a new class of radio transient. FRBs are characterized by millisecond durations, $\sim$ Jansky flux densities, and dispersion measures (DMs) consistent with sources at gigaparsec $(\mathrm{Gpc})$ distances. Now observed with a growing number of instruments, including the Parkes (Lorimer et al. 2007; Keane et al. 2011; Thornton et al. 2013; Burke-Spolaor \& Bannister 2014; Petroff et al. 2015; Ravi et al. 2015; Champion et al. 2016; Keane et al. 2016), Arecibo (Spitler et al. 2014, 2016), and Green Bank (Masui et al. 2015) telescopes, FRBs are becoming increasingly accepted as a true astronomical phenomenon, rather than local signals of terrestrial origin. Recently, Keane et al. (2016) have even reported the first identification of an FRB's host galaxy, although this claim is currently disputed (Vedantham et al. 2016; Williams \& Berger 2016). FRBs also appear to be quite numerous. While only 17 FRBs have been reported to date (Petroff et al. 2016; see the FRBCAT $^{1}$ ), after correcting for sky coverage and observing cadence it is estimated that between $10^{3}$ and $10^{4}$ occur on the sky per day (Thornton et al. 2013; Keane \& Petroff 2015). That is, a hypothetical telescope array observing continuously with complete sky coverage would observe between 1000 and 10,000 FRBs per day.

A large number of theories have been put forward as to the possible source(s) of FRBs. Theorized sources include (but are certainly not limited to) the collapse of supramassive neutron stars (Falcke \& Rezzolla 2014; Ravi \& Lasky 2014; Zhang 2014), supergiant neutron star pulses (Connor et al. 2016; Cordes \& Wasserman 2016), pulsar-planet systems (Mottez \& Zarka 2014), bremmstrahlung from gamma-ray bursts or active galactic nuclei (Romero et al. 2016), and galactic flare stars (Loeb et al. 2014; Maoz et al. 2015). More exotic sources include the explosions of white holes (Barrau et al. 2014) and primordial black hole evaporation (Keane et al. 2012).

Compact binary coalescences (CBCs) represent another broad class of theorized FRB progenitor. The mergers of binary neutron stars (BNSs; Totani 2013; Wang et al. 2016),

\footnotetext{
http://www.astronomy.swin.edu.au/pulsar/frbcat/
}

neutron star-black hole (NSBH) binaries (Mingarelli et al. 2015), white dwarf binaries (Kashiyama et al. 2013), and charged binary black holes (BBHs; Liu et al. 2016; Zhang 2016a) have all been put forward as possible sources of FRB emission. Furthermore, the recent localization of FRB 150418 to an elliptical galaxy with low star formation would, if correct, support a CBC progenitor (Keane et al. 2016).

The possibility that binary coalescences are FRB progenitors is particularly attractive. If this were indeed the case, then FRBs would be promising electromagnetic counterparts to gravitational-wave detections of compact binary mergers, and would be valuable targets for future multi-messenger studies (Kaplan et al. 2015; Yancey et al. 2015). The recent discovery of the repeating fast radio burst FRB 121102 (Spitler et al. 2016) points to a non-cataclysmic origin for at least some fraction of FRBs, ruling out binary coalescences as the sole progenitors of all FRBs. As has been pointed out, though, FRBs may not constitute a single population (Mingarelli et al. 2015; Spitler et al. 2016); there may instead exist multiple FRB populations, each arising from a different class of progenitor. If binary coalescences are to be considered plausible models for one such progenitor population, then their astrophysical rates must be consistent with the inferred rate of FRBs.

In this Letter, we demonstrate that existing and future gravitational-wave measurements of the rates of binary coalescences can be leveraged to place novel constraints on the nature of FRB progenitors. In some cases, we can confidently rule out certain classes of binary coalescences as dominant FRB progenitors.

\section{RATES OF COMPACT BINARY COALESCENCES}

The recent Advanced LIGO and Virgo detection of the BBH merger GW150914 (Abbott et al. 2016a) has produced the first direct measurement of the $\mathrm{BBH}$ merger rate per comoving volume (the so-called "rate density") in the nearby universe. From this event, it is inferred that the BBH merger rate density lies between 2 and $400 \mathrm{Gpc}^{-3} \mathrm{yr}^{-1}$ (Abbott et al. 2016c).

While the rate densities of BNS and NSBH mergers remain unknown, binary pulsar observations and population synthesis 


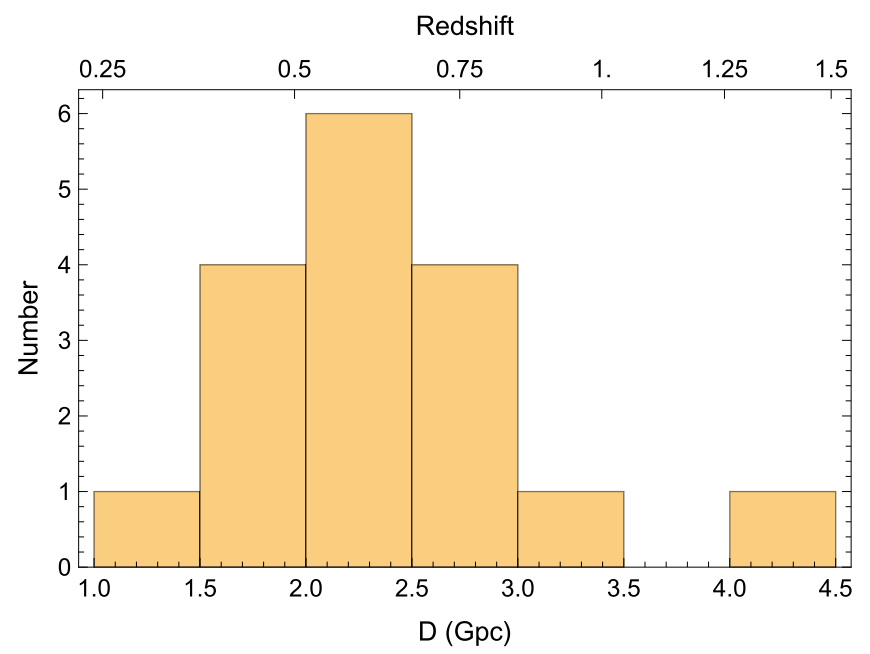

Figure 1. Distribution of inferred distances to known FRBs, assuming a homogeneous, fully ionized intergalactic medium and neglecting dispersion measure contributions from both the Milky Way and FRB host galaxies. We take $3 \mathrm{Gpc}$ as a fiducial distance bounding the observed FRB population.

models place rough bounds on the expected BNS and NSBH rates, respectively. BNS and $\mathrm{NSBH}$ merger rate densities are predicted to plausibly fall between $R_{\mathrm{BNS}}=10-10^{4} \mathrm{Gpc}^{-3} \mathrm{yr}^{-1}$ and $R_{\mathrm{NSBH}}=0.6-10^{3} \mathrm{Gpc}^{-3} \mathrm{yr}^{-1}$ (Kalogera et al. 2004; O'Shaughnessy et al. 2008; Abadie et al. 2010; Kim et al. 2015). Note, however, that Dominik et al. (2013) predict NSBH rate densities as low as $0.04 \mathrm{Gpc}^{-3} \mathrm{yr}^{-1}$. Gravitationalwave experiments have not yet begun to probe these predicted ranges; the best experimental results, placed jointly by Initial LIGO and Initial Virgo, limit BNS and NSBH merger rate densities to $R_{\mathrm{BNS}}<1.3 \times 10^{5} \mathrm{Gpc}^{-3} \mathrm{yr}^{-1}$ and $R_{\mathrm{NSBH}}<$ $3.1 \times 10^{4} \mathrm{Gpc}^{-3} \mathrm{yr}^{-1}$, respectively (Abadie et al. 2012).

Although the Initial LIGO/Virgo limits are well above the most optimistic predictions from population synthesis and binary pulsars, Advanced LIGO's recently concluded first observing run $(\mathrm{O} 1)$ is expected to measure rate densities down to $R_{\mathrm{BNS}} \approx 3 \times 10^{3} \mathrm{Gpc}^{-3} \mathrm{yr}^{-1}$ and $R_{\mathrm{NSBH}} \approx 750 \mathrm{Gpc}^{-3} \mathrm{yr}^{-1}$, experimentally probing for the first time the range of astrophysically plausible merger rates (Abbott et al. 2016b). In 2017-18, Advanced LIGO's second observing run (O2) is projected to be sensitive to rate densities as low as $R_{\mathrm{BNS}} \approx$ $450 \mathrm{Gpc}^{-3} \mathrm{yr}^{-1}$ and $R_{\mathrm{NSBH}} \approx 100 \mathrm{Gpc}^{-3} \mathrm{yr}^{-1}$, while the its third run $(\mathrm{O} 3 ; 2018-19)$ further pushes Advanced LIGO's sensitivity to $R_{\mathrm{BNS}} \approx 100 \mathrm{Gpc}^{-3} \mathrm{yr}^{-1}$ and $R_{\mathrm{NSBH}} \approx 20 \mathrm{Gpc}^{-3} \mathrm{yr}^{-1}$.

\section{RATES OF FRBs}

The predicted and measured rates of binary coalescences allow for direct constraints on the nature of FRB progenitors by comparison to the inferred FRB rate per comoving volume. Other authors have considered the physical rate of FRBs, but these calculations are typically not shown in detail and significant disagreement exists in the literature, e.g., Totani (2013) versus Zhang (2016a, 2016b). Our goal in this section is therefore a careful accounting of the FRB rate density. As we will show below, the FRB rate per comoving volume is potentially far higher than the corresponding rate densities of binary coalescences. Thus, it is unlikely that the coalescence of stellar-mass compact binaries represents more than a small fraction of FRB progenitors. Because of this rate discrepancy, the lowest $\mathrm{FRB}$ rate estimates are most compatible with $\mathrm{CBC}$ progenitors. In the following, we will therefore deliberately seek a lower limit on the FRB rate density in order to most generously assess the plausibility of CBC progenitors of FRBs.

The inferred FRB rate per comoving volume is approximately $\left(3 r_{\text {obs }}\right) /\left(4 \pi D^{3}\right)$. Here, $D$ is the comoving distance containing the observed FRB population and $r_{\mathrm{obs}}$ is the observed rate at which FRBs occur on the sky. For simplicity, we will assume this rate density is constant and neglect evolution with redshift. If FRB emission is beamed, then the rate $r_{\mathrm{obs}}$ is undercounted due to selection effects-beamed FRBs, like pulsars or GRBs, are only observed if the Earth lies within the path of the beam. In general, the FRB rate per comoving volume is

$$
R_{\mathrm{FRB}} \approx \frac{3 r_{\mathrm{obs}}}{\Omega D^{3}}
$$

where $\Omega$ is a typical solid angle over which emission is beamed.

Although few FRBs have been observed, their inferred rate on the sky is large. With four FRB detections at high Galactic latitudes using Parkes, Thornton et al. (2013) inferred that $r_{\text {obs }}=1.0_{-0.5}^{+0.6} \times 10^{4}$ FRBs occur on the sky per day. However, there remains considerable disagreement as to the true value of $r_{\text {obs }}$, with subsequent radio surveys producing differing rate estimates, often defined with respect to different fluence limits and different assumptions about search systematics.

Keane \& Petroff (2015), for instance, point out that FRB detection is subject to significant selection effects, such as survey incompleteness below a fluence of $\sim 2 \mathrm{Jy} \mathrm{ms,} \mathrm{subopti-}$ mal recovery of broad radio pulses, and potential obscuration of FRBs in the galactic plane. They estimate a fluence-limited detectable FRB rate of $2500 \mathrm{sky}^{-1} \mathrm{day}^{-1}$ above $\sim 2 \mathrm{Jy} \mathrm{ms}$. Macquart \& Johnston (2015) also arrive at $r_{\text {obs }} \approx$ $2500 \mathrm{sky}^{-1}$ day $^{-1}$ but by different means, suggesting that the apparent FRB rate at high latitudes is enhanced by interstellar scintillation. Rane et al. (2016) adopt a Bayesian approach, combining several published rate estimates to obtain $r_{\text {obs }}=4.4_{-3.1}^{+5.2} \times 10^{3} \mathrm{sky}^{-1} \mathrm{day}^{-1}$ above $4.0 \mathrm{Jy} \mathrm{ms}$. On the other hand, Law et al. (2015) argue that previously published singledish rate estimates are biased below their true values and that, once potential biases are corrected, previous estimates are consistent with $r_{\text {obs }}=1.2 \times 10^{4} \mathrm{sky}^{-1} \mathrm{day}^{-1}$ above $1.7 \mathrm{Jy} \mathrm{ms}$.

It is not obvious which value to select for $r_{\text {obs }}$ (or even which range of uncertainties to adopt). In order to place a lower limit on the FRB rate density, however, we will take $r_{\mathrm{obs}}=2500 \mathrm{sky}^{-1} \mathrm{day}^{-1}$, consistent with the lowest of the above estimates. To additionally allow for various search selection effects, we will define $\eta$ as the FRB detection efficiency, the fraction of otherwise detectable FRBs (e.g., with intrinsic signal-to-noise ratios above some threshold detection value) that are actually recovered in a radio transient search. The physical rate of FRBs on the sky is then $r_{\mathrm{obs}} /(\eta \Omega)$.

Distances to FRB sources may be estimated using their reported DMs, which we obtained from the FRBCAT (Petroff et al. 2016). Assuming that the intergalactic medium (IGM) is homogeneous and fully ionized, the dispersion measure $\mathrm{DM}_{\mathrm{IGM}}$ due to propagation through the IGM is related to source redshift via (Ioka 2003; Inoue 2004)

$$
\operatorname{DM}_{\mathrm{IGM}}(z)=\frac{\bar{n}_{e} c}{H_{0}} \int_{0}^{z} \frac{\left(1+z^{\prime}\right) d z^{\prime}}{\sqrt{\Omega_{\mathrm{M}}\left(1+z^{\prime}\right)^{3}+\Omega_{\Lambda}}},
$$


where $\bar{n}_{e}=\rho_{c} \Omega_{\mathrm{B}} / m_{p}=2.5 \times 10^{-7} \mathrm{~cm}^{-3}$ is the local free electron density in a fully ionized universe. Here, $\Omega_{\mathrm{B}}, \Omega_{\mathrm{M}}$, and $\Omega_{\Lambda}$ are the energy densities of baryons, matter, and dark energy, respectively, $m_{p}$ is the proton mass, and $\rho_{c}=3 H_{0}^{2} / 8 \pi G$ is the critical energy density required to close the universe. $G$ is Newton's constant, $c$ is the speed of light, and $H_{0}$ is the Hubble constant; we use the Planck Collaboration et al. (2015) parameters $H_{0}=67.7 \mathrm{~km} \mathrm{~s}^{-1} \mathrm{Mpc}^{-1}, \Omega_{\mathrm{B}}=0.049$, $\Omega_{\mathrm{M}}=0.31$, and $\Omega_{\Lambda}=0.69$. In the small redshift limit, Equation (2) reduces to $\mathrm{DM}_{\mathrm{IGM}}(z) \approx \bar{n}_{e} D$, where $D=c z / H_{0}$ is the approximate source distance at low redshifts. In general, comoving distance is given by

$$
D(z)=\frac{c}{H_{0}} \int_{0}^{z} \frac{d z^{\prime}}{\sqrt{\Omega_{\mathrm{M}}\left(1+z^{\prime}\right)^{3}+\Omega_{\Lambda}}} .
$$

Using Equations (2) and (3), the inferred comoving distances to the 17 known FRBs are shown in Figure 1. Based on this sample, we will take $D=3 \mathrm{Gpc}$ as a fiducial distance encompassing the observed FRB population.

We have made several assumptions in computing the distances shown in Figure 1. Since a factor of 2 error in the fiducial distance will result in a factor of $2^{3}$ error in the FRB rate density, it is important to highlight these assumptions and understand how they affect our result. First, we assumed that the observed radio dispersions are entirely due to propagation through the IGM. In reality, the Milky Way may contribute up to $20 \%$ of the observed DM (Petroff et al. 2016). The distances in Figure 1 may therefore be overestimated by a factor of $\sim 1.25$. If we also allow for a comparable DM contribution by the FRB's host galaxy (as well contributions from any matter overdensities along the line of sight to the FRB), then the distances may be overestimated by at least a factor of 1.7. This implies that our FRB rate density is underestimated by a factor between 2 and 5 .

Second, we assumed a fully ionized universe. While valid for hydrogen, this is not necessarily true for helium, which may be either singly or fully ionized. Helium makes up approximately $24 \%$ of the IGM by mass (Inoue 2004); if this helium is only singly ionized, then the free electron density $\bar{n}_{e}$ will be reduced by roughly $10 \%$. Finally, $\Omega_{\mathrm{B}}$ is an overestimate of the baryon density in the IGM, since $\sim 10 \%$ of baryons are sequestered in galaxies (Fukugita \& Peebles 2004). Together, these two approximations cause $\bar{n}_{e}$ to be (at most) $20 \%$ larger than the true free electron density in the IGM. By overestimating $\bar{n}_{e}$, the fiducial distance $D$ is underestimated by a factor of 1.25 , and the FRB rate density is overestimated by a factor of 2 .

Note that, of the assumptions described here, the first (uncertainty in the intergalactic DM) will cause the fiducial distance to be underestimated, while the second and third (uncertainty in $\bar{n}_{e}$ ) cause the distance to be overestimated. Of these uncertainties, the potentially large overestimate of the intergalactic DM is expected to dominate. Thus our choice of $D=3 \mathrm{Gpc}$ is likely an upper bound on the fiducial FRB distance. Because $R_{\mathrm{FRB}} \propto D^{-3}$, any decrease in the fiducial distance will only increase the FRB rate density, further increasing the tension between the rates of FRBs and binary coalescences.

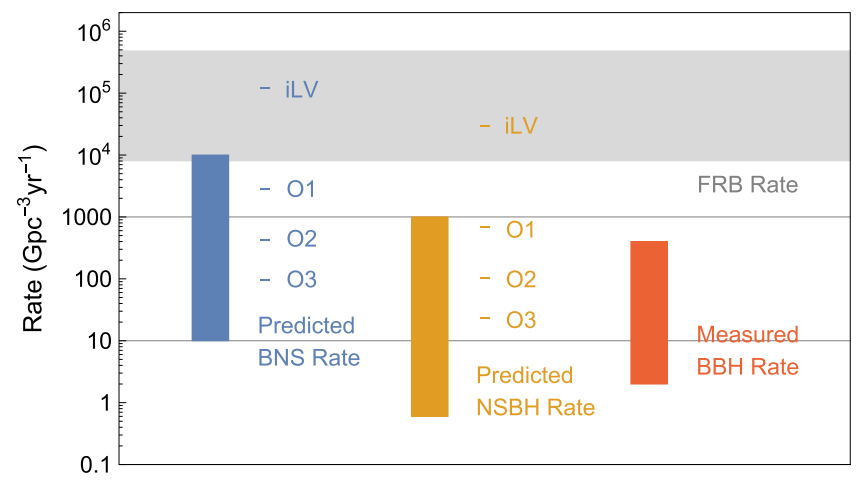

Figure 2. Binary coalescence rates compared to the inferred rate of FRBs. Solid bars indicate the range of BNS (blue) and NSBH (orange) merger rates predicted by binary pulsar observations and population synthesis models (Abadie et al. 2010), as well as the measured LIGO/Virgo rate of BBH mergers (red; Abbott et al. 2016c). Also shown are existing Initial LIGO/Virgo (iLV) limits and projected $\mathrm{O} 1, \mathrm{O} 2$, and $\mathrm{O} 3$ sensitivities (Abadie et al. 2012; Abbott et al. 2016b). The gray band indicates a range of potential FRB rate densities, from the lowest plausible value in Equation (5) to a more realistic estimate in Equation (6).

All together, the FRB rate per comoving volume is

$$
\begin{aligned}
R_{\mathrm{FRB}}= & \left(8.1 \times 10^{3} \mathrm{Gpc}^{-3} \mathrm{yr}^{-1}\right)\left(\frac{r_{\mathrm{obs}}}{2500 \mathrm{sky}^{-1} \mathrm{day}^{-1}}\right) \\
& \times\left(\frac{1}{\eta}\right)\left(\frac{3 \mathrm{Gpc}}{D}\right)^{3}\left(\frac{4 \pi \mathrm{Sr}}{\Omega}\right) .
\end{aligned}
$$

The lowest plausible FRB rate density, corresponding to $r_{\text {obs }}=2500 \mathrm{sky}^{-1}$ day $^{-1}$, radio transient detection efficiencies of $\eta=1$, and isotropic radio emission $(\Omega=4 \pi \mathrm{Sr})$, is

$$
R_{\mathrm{FRB}}^{\text {Low }}=8.1 \times 10^{3} \mathrm{Gpc}^{-3} \mathrm{yr}^{-1} \text {. }
$$

A more realistic rate density, on the other hand, is obtained by assuming $r_{\mathrm{obs}}=5000 \mathrm{sky}^{-1} \mathrm{day}^{-1}$, a detection efficiency of $\eta=0.5$, and beamed emission with a $30^{\circ}$ half-opening angle. These values give

$$
R_{\mathrm{FRB}}^{\text {Realistic }}=4.8 \times 10^{5} \mathrm{Gpc}^{-3} \mathrm{yr}^{-1},
$$

nearly two orders of magnitude larger than Equation (5).

Our lower limit on the FRB rate density agrees well with the rate previously estimated by Totani (2013). It is, however, more than an order of magnitude higher than the more recent result computed by Zhang (2016a, 2016b). The discrepancy lies in the fact that Zhang (2016a, 2016b) improperly uses the luminosity distance $D_{L}=D(1+z)$ rather than the comoving distance to calculate the FRB rate density. Zhang (2016a, 2016b) chooses $z=1$ as a fiducial redshift, at which $D=3.4 \mathrm{Gpc}$ while $D_{L}=6.8 \mathrm{Gpc}$. This factor of 2 adjustment in distance leads to a factor of 8 discrepancy in the FRB rate density. Using $D=3.4 \mathrm{Gpc}$ in Equation (8) of Zhang (2016b) gives an FRB rate density of $5.8 \times 10^{3} \mathrm{Gpc}^{-3} \mathrm{yr}^{-1}$, in reasonably good agreement with our lower limit.

\section{COMPACT BINARIES AS FRB PROGENITORS?}

By comparing $R_{\mathrm{FRB}}$ from Section 3 to the binary coalescence rates in Section 2, we can constrain the fraction of FRBs that can be explained via compact binary coalescences. Figure 2 shows a range of potential FRB rate densities, from the lowest plausible estimate given in Equation (5) (assuming 
$r_{\mathrm{obs}}=2500 \mathrm{sky}^{-1} \mathrm{day}^{-1}$, efficiency $\eta=1$, and isotropic FRB emission) to the more realistic value in Equation (6) (which assumes $r_{\mathrm{obs}}=5000 \mathrm{sky}^{-1} \mathrm{day}^{-1}$, efficiency $\eta=0.5$, and FRB beaming with a half-opening angle of $30^{\circ}$ ). Solid bars indicate the range of BNS and NSBH merger rate densities predicted by binary pulsar observations and population synthesis models, as well as the measured $\mathrm{BBH}$ rate density. Also shown are existing Initial LIGO/Virgo limits, as well as the projected sensitivities of the $\mathrm{O} 1, \mathrm{O} 2$, and $\mathrm{O} 3$ observing runs.

Binary black holes: the measured rate of $\mathrm{BBH}$ mergers is at most $\sim 5 \%$ of the inferred FRB rate. Thus, BBHs cannot explain more than a small fraction of the observed FRB population. Previous claims that the rates of FRBs and BBH mergers are consistent (Zhang 2016a, 2016b) are based on an erroneous calculation of the FRB rate density, as discussed in Section 3.

Neutron star-black hole binaries: population synthesis predictions are highly inconsistent with the theory that NSBH mergers are FRB progenitors, with predicted NSBH merger rates equal to at most $\sim 12 \%$ of the FRB rate. This fraction assumes isotropic radio emission, and hence should be taken as a highly optimistic upper limit on the FRB fraction compatible with NSBH binaries. Even moderate beaming, with a halfopening angle of e.g., $30^{\circ}$, reduces the predicted FRB fraction to $\sim 0.8 \%$. Assuming the realistic FRB rate density in Equation (6) further lowers this fraction by a factor of four.

Although Initial LIGO/Virgo upper limits are uninformative (limiting the most optimistic NSBH fraction of FRB progenitors to $R_{\mathrm{NSBH}} / R_{\mathrm{FRB}}^{\text {Low }} \lesssim 4$ ), Advanced LIGO is capable of measuring significantly smaller NSBH merger rates. A nondetection during the $\mathrm{O} 1$ and $\mathrm{O} 2$ observing runs, for instance, would limit the NSBH FRB fraction to $\lesssim 9 \%$ and $\lesssim 1 \%$, respectively (assuming isotropic emission).

Binary neutron stars: there exist competing claims as to whether the rates of FRBs and BNS mergers are (Totani 2013; Wang et al. 2016) or are not (Thornton et al. 2013) compatible. ${ }^{2}$ We find that the most optimistic BNS rate density predictions are consistent with the lowest possible FRB rate density, with $R_{\mathrm{BNS}} / R_{\mathrm{FRB}}^{\text {low }} \approx 1.2$ Therefore, BNS mergers could constitute a subpopulation of FRB progenitor if multiple FRB subclasses do indeed exist. This compatibility is tenuous, however, simultaneously requiring the highest possible BNS rates and the lowest possible FRB rates (with, e.g., perfect FRB detection efficiency and isotropic radio emission). FRB models that predict even moderately beamed emission are largely incompatible with BNS progenitors.

If BNS mergers are indeed FRB progenitors, then it is likely that Advanced LIGO will observe a large number of BNS sources in the $\mathrm{O} 1$ observing run. If no such detections are made, then the resulting rate limits will increasingly cast doubt on the role of BNSs as FRB progenitors. An Advanced LIGO non-detection during $\mathrm{O} 1$ and $\mathrm{O} 2$ would limit the most optimistic fraction of FRBs compatible with BNS mergers to $\lesssim 40 \%$ and $\lesssim 6 \%$, respectively. If we assume moderate FRB beaming (again with a half-opening angle of $30^{\circ}$ ), then $\mathrm{O} 1$ and $\mathrm{O} 2$ non-detections imply even more stringent FRB fractions of $\lesssim 2 \%$ and $\lesssim 0.4 \%$, respectively. Note that these limits also apply equally to short-lived products of BNS mergers, such as hypermassive neutron stars.

\footnotetext{
2 Note that Wang et al. (2016) adopt the FRB rate estimate of Zhang (2016b), and hence incorrectly find strong consistency between the rates of BNS mergers and FRBs.
}

\section{CONCLUSIONS}

A diverse range of FRB progenitor models have been proposed, including the binary coalescences of neutron stars and/or black holes. Existing or future limits from gravitationalwave observations can serve to severely constrain such models. The recent Advanced LIGO/Virgo measurement of the local BBH merger rate density largely rules out stellar-mass BBHs as progenitors of the observed FRB population. Meanwhile, predictions of NSBH merger rate densities from population synthesis are in strong tension with the inferred rate density of FRBs; upcoming observations by Advanced LIGO and Virgo could rule out NSBHs as FRB progenitors.

Under generous assumptions (broadly beamed radio emission, large FRB distances, and low underlying FRB rates), the rate of BNS mergers may be consistent with a subpopulation of FRB progenitors. In order for this subpopulation to be significant, however, the BNS merger rate density must be on the order of $\sim 10^{4} \mathrm{Gpc}^{-3} \mathrm{yr}^{-1}$, comparable to the most optimistic predictions from population synthesis. Additionally, FRB emission must be largely isotropic; models that predict even moderately beamed emission are inconsistent with BNS rates. If BNS mergers are indeed FRB progenitors, then Advanced LIGO and Virgo will soon begin to observe a large number of such systems. If no such observations are made, then the resulting rate limits will increasingly constrain the ability of BNSs to explain even a subclass of the FRB population.

We thank Krzysztof Belczynski, Christopher Berry, Daniel Hoak, Brennan Hughey, Shri Kulkarni, and Graham Woan, as well as the anonymous referee whose comments greatly improved this text. The authors are members of the LIGO Laboratory, supported by the United States National Science Foundation. LIGO was constructed by the California Institute of Technology and Massachusetts Institute of Technology with funding from the National Science Foundation and operates under cooperative agreement PHY-0757058. This paper carries LIGO Document Number P1600106. A Mathematica notebook that reproduces the results in this paper is available at: https:// github.com/tcallister/GravWaveFRBConstraints.

Software: Mathematica.

\section{REFERENCES}

Abadie, J., Abbott, B. P., Abbott, R., et al. 2010, CQGra, 27, 173001 Abadie, J., Abbott, B. P., Abbott, R., et al. 2012, PhRvD, 85, 082002 Abbott, B. P., Abbott, R., Abbott, T. D., et al. 2016a, PhRvL, 116, 061102 Abbott, B. P., Abbott, R., Abbott, T. D., et al. 2016b, LRR, 19, 1 Abbott, B. P., Abbott, R., Abbott, T. D., et al. 2016c, arXiv:1602.03842 Barrau, A., Rovelli, C., \& Vidotto, F. 2014, PhRvD, 90, 127503 Burke-Spolaor, S., \& Bannister, K. W. 2014, ApJ, 792, 19 Champion, D. J., Petroff, E., Kramer, M., et al. 2016, MNRAS, 460, L30 Connor, L., Sievers, J., \& Pen, U.-L. 2016, MNRAS, 458, L19

Cordes, J. M., \& Wasserman, I. 2016, MNRAS, 457, 232

Dominik, M., Belczynski, K., Fryer, C., et al. 2013, ApJ, 779, 72

Falcke, H., \& Rezzolla, L. 2014, A\&A, 562, A137

Fukugita, M., \& Peebles, P. J. E. 2004, ApJ, 616, 643

Inoue, S. 2004, MNRAS, 348, 999

Ioka, K. 2003, ApJL, 598, L79

Kalogera, V., Kim, C., Lorimer, D. R., et al. 2004, ApJL, 614, L137

Kaplan, D. L., Rowlinson, A., Bannister, K. W., et al. 2015, ApJL, 814, L25

Kashiyama, K., Ioka, K., \& Mészáros, P. 2013, ApJL, 776, L39

Keane, E. F., Johnston, S., Bhandari, S., et al. 2016, Natur, 530, 453

Keane, E. F., Kramer, M., Lyne, A. G., Stappers, B. W., \& McLaughlin, M. A. 2011, MNRAS, 415, 3065

Keane, E. F., \& Petroff, E. 2015, MNRAS, 447, 2852 
Keane, E. F., Stappers, B. W., Kramer, M., \& Lyne, A. G. 2012, MNRAS, 425, L71

Kim, C., Perera, B. B. P., \& McLaughlin, M. a. 2015, MNRAS, 448, 928

Law, C. J., Bower, G. C., Burke-Spolaor, S., et al. 2015, ApJ, 807, 16

Liu, T., Romero, G. E., Liu, M.-L., \& Li, A. 2016, ApJ, in press (arXiv:1062. 06907)

Loeb, A., Shvartzvald, Y., \& Maoz, D. 2014, MNRAS, 439, L46

Lorimer, D. R., Bailes, M., McLaughlin, M. A., Narkevic, D. J., \& Crawford, F. 2007, Sci, 318, 777

Macquart, J.-P., \& Johnston, S. 2015, MNRAS, 451, 3278

Maoz, D., Loeb, A., Shvartzvald, Y., et al. 2015, MNRAS, 454, 2183

Masui, K., Lin, H.-H., Sievers, J., et al. 2015, Natur, 528, 523

Mingarelli, C. M. F., Levin, J., \& Lazio, T. J. W. 2015, ApJL, 814, L20

Mottez, F., \& Zarka, P. 2014, A\&A, 569, A86

O’Shaughnessy, R., Kim, C., Kalogera, V., \& Belczynski, K. 2008, ApJ, 672, 479

Petroff, E., Bailes, M., Barr, E. D., et al. 2015, MNRAS, 447, 246

Petroff, E., Barr, E. D., Jameson, A., et al. 2016, arXiv:1601.03547
Planck Collaboration, Ade, P. A. R., Aghanim, N., et al. 2015, arXiv:1502. 01589

Rane, A., Lorimer, D. R., Bates, S. D., et al. 2016, MNRAS, 455, 2207

Ravi, V., \& Lasky, P. D. 2014, MNRAS, 441, 2433

Ravi, V., Shannon, R. M., \& Jameson, A. 2015, ApJL, 799, L5

Romero, G. E., del Valle, M. V., \& Vieyro, F. L. 2016, PhRvD, 93, 023001

Spitler, L. G., Cordes, J. M., Hessels, J. W. T., et al. 2014, ApJ, 790, 101

Spitler, L. G., Scholz, P., Hessels, J. W. T., et al. 2016, Natur, 531, 202

Thornton, D., Stappers, B., Bailes, M., et al. 2013, Sci, 341, 53

Totani, T. 2013, PASJ, 65, L12

Vedantham, H. K., Ravi, V., Mooley, K., et al. 2016, ApJL, 824, L9

Wang, J.-S., Yang, Y.-P., Wu, X.-F., Dai, Z.-G., \& Wang, F.-Y. 2016, ApJL, 822, L7

Williams, P. K. G., \& Berger, E. 2016, ApJL, 821, L22

Yancey, C. C., Bear, B. E., Akukwe, B., et al. 2015, ApJ, 812, 168

Zhang, B. 2014, ApJL, 780, L21

Zhang, B. 2016a, arXiv: 1602.04542

Zhang, B. 2016b, ApJL, 822, L14 